Die Beratung des Präventionsgesetzes geht in die nächste Runde: Die Gesundheitskommission des Nationalrates hat die Eckpfeiler gesetzt, nun ist es an den Räten, der Schweiz ein Präventionsgesetz zu geben, das den neuen gesundheitlichen Herausforderungen gerecht wird. Die Kommission hat festgehalten, dass das Präventionsgesetz zur Reduktion gesundheitlicher Ungleichheiten beitragen soll. Damit hat sie eine wichtige Weiche gestellt:
Die Gesundheitschancen sind ungleich verteilt - Prävention ist dann am erfolgreichsten, wenn sie an diesem Hebel ansetzt. Es wird Zeit, dass die eidgenössischen Räte nun die gesetzlichen Grundlagen dazu schaffen.

Dr. med. Christine Romann, Mitglied des Zentralvorstands der FMH, Verantwortliche Ressort Gesundheitsförderung und Prävention

\title{
Präventionsgesetz: modern times
}

Moderne Gesundheitsförderung und Prävention findet dort statt, wo Menschen leben, arbeiten, spielen, lernen - in ihrem Quartier, im Kindergarten, in der Schule, am Arbeitsplatz oder in der Freizeit.

Die aktuelle Schweizer Gesetzgebung regelt Gesundheitsprobleme des 19. Jahrhunderts mit den Massnahmen vergangener Zeiten. Abgesehen von der Bekämpfung übertragbarer Krankheiten, der Verhütung von Sucht- und Berufskrankheiten und der Unfallprävention sind keine gesetzlichen Grundlagen vorhanden. Das gilt insbesondere für nicht übertragbare und psychische Krankheiten. Doch es sind gerade diese Bereiche, welche in unserer Zeit die höchsten Kosten verursachen.

\section{Mit dem neuen Präventionsgesetz haben wir die Chance, endlich zeitgemässe nationale Gesund- heitsziele zu definieren.}

Heute entfällt der grösste Teil der Krankheitslast auf nicht übertragbare Krankheiten - eine Gruppe von Erkrankungen, zu welcher Herz-Kreislauf-Erkrankungen, Krebs, psychische Gesundheitsprobleme, Diabetes mellitus, chronische Atemwegserkrankungen oder muskuloskelettale Erkrankungen gehören. Auf dieses breite Spektrum an Störungen, die gemeinsame Risikofaktoren, Determinanten und Interventionsmöglichkeiten aufweisen, entfallen $86 \%$ der Todesfälle und $77 \%$ der Krankheitslast in der Europäischen Region der WHO. Innerhalb unserer Gesellschaft sind die verschiedenen Erkrankungen und ihre Ursachen insgesamt ungleich verteilt: Angehörige von Gruppen mit niedrigem sozioökonomischen Status tragen ein mindestens doppelt so hohes Risiko, ernsthaft zu erkranken und vorzeitig zu sterben, wie Angehörige privilegierter Schichten. Und auch dort, wo es zu Verbesserungen der Gesundheitssituation kommt, ist der Nutzen innerhalb der Gesellschaft ungleich verteilt: Angehörige von Gruppen mit höherem sozioökonomischen Status reagieren oft positiver und ziehen grösseren Nutzen.

Nach Einschätzung vieler Experten wird das gesundheitspolitische und gesundheitsökonomische Potential von Gesundheitsförderung und Prävention noch immer zu wenig wahrgenommen. Aktivitäten - zum Beispiel im Bereich Umwelt, Arbeit, Bildung und Ernährung - haben nach internationalen Erkenntnissen einen entscheidenderen Anteil an der Erhaltung und Verbesserung des Gesundheitszustandes als medizinisch-kurative Leistungen. So ist es erwiesen, dass Massnahmen zu Gesundheitsförderung und Prävention kosteneffektiv und kostensparend sind. Zum Beispiel beugen bei Kindern 30 Minuten Bewegung pro Tag Übergewicht vor. Oder sie entwickeln ein besseres Sozialverhalten, wenn sie musisch gefördert werden.

Mit dem neuen Präventionsgesetz haben wir die Chance, endlich zeitgemässe nationale Gesundheitsziele zu definieren, eine kantonsübergreifende Strategie für Gesundheitsförderung und Prävention zu erarbeiten. Bisherige Strukturen werden vereinfacht, bestehende Aktivitäten besser koordiniert und damit finanzielle Mittel effizienter eingesetzt. Das neue Präventionsgesetz wird für Klarheit bei den Zuständigkeiten und bei der Finanzierung der Präventionstätigkeiten sorgen.

Das Einstehen für Schwächere ist seit jeher zentraler Teil unseres Selbstverständnisses, die Standesordnung der Schweizer Ärzteschaft legt es dar: «Es ist Aufgabe des Arztes und der Ärztin, menschliches Leben zu schützen, Gesundheit zu fördern und zu erhalten.» Auch die tägliche Praxis des Arztes hat sich in den letzten 30 Jahren verändert - vom paternalisti-

\section{Die Zukunft liegt nicht in Einzellösungen, sondern in übergreifenden Ansätzen, die den Menschen ins Zentrum stellen.}

schen Heiler zum partnerschaftlichen Begleiter und Betreuer seiner Patienten. Ärztinnen und Ärzte kennen sehr wohl die Bedeutung der Umwelt, Arbeit und sozialen Sicherheit für die Gesundheit ihrer Patienten. Die Zukunft liegt nicht in Einzellösungen für Herz, Hirn oder Lunge, sondern in übergreifenden Ansätzen, die den Menschen ins Zentrum stellen. Tempora mutantur nos et mutamur in illis.

Barbara Weil, Leiterin Abteilung Gesundheitsförderung und Prävention der FMH 